\title{
Tuberculous Pleuro-Pericardial Effusion with Adenitis
}

\author{
Adeyeye $\mathrm{VO}^{1^{*}}$, Aramide $\mathrm{KO}^{2}$, Adefalujo $\mathrm{AP}^{3}$ and Ayodele $\mathrm{OO}^{1}$ \\ ${ }^{1}$ Department of Medicine, Babcock University Teaching Hospital, Ilishan-Remo, Ogun State, Nigeria \\ ${ }^{2}$ Department of Histopathology, Babcock University Teaching Hospital, Ilishan-Remo, Ogun State, Nigeria \\ ${ }^{3}$ Department of Radiology, Babcock University Teaching Hospital, Ilishan-Remo, Ogun State, Nigeria
}

"Corresponding Author: Adeyeye VO, Department of Medicine, Babcock University Teaching Hospital, IlishanRemo, Ogun State, Nigeria, Tel: +2348067651916; Email: vioyeye@ yahoo.com

Received: 13 August 2017; Accepted: 24 August 2017; Published: 30 August 2017

\begin{abstract}
Background: Hydra-headed clinical presentations of tuberculosis could be confusing even in immunocompetent, for example pleuro-pericardial effusion with adenitis. From our literature search, this is rare.

Case presentation: A 61-year-old clergyman presented with a 3-week history of cough, fever and a 5-day history of progressive dyspnoea. There was associated anaemia, weight loss but no history of drenching night sweat, hemoptysis, exposure to tuberculosis, diabetes or other suggestion of immunosuppression. Examination revealed multiple matted left axillary lymph nodes. Erythrocyte sedimentation rate (ESR) was $70 \mathrm{~mm} / \mathrm{hr}$; and negative for Hepatitis B, C and HIV. Chest X-ray showed left lower lung zone homogenous opacity with obliteration of the left cardiac border. Lymph node aspirate and pleural fluid analyses confirmed a tuberculous aetiology.
\end{abstract}

Conclusion: Tuberculous pleuro-pericardial effusion with adenitis occurs in immunocompetent. High index of suspicion in endemic areas as well as early anti-tubercular treatment could be life-saving.

Keywords: Clergyman; Effusion; Pleuro-pericardial; Tuberculosis

\section{Introduction}

Tuberculosis (TB) remains a clinical challenge in developing countries; be its diagnosis, treatment and prevention (primary and secondary). Globally, incidence of TB is increasing due to HIV pandemic, multi-drug resistance and excruciating socio-economic melt-down. Africa, Asia and Latin America account for $98 \%$ of nearly two million deaths resulting from tuberculosis each year [1]. Its clinical presentations could be hydra-headed. Up to $4 \%$ of cases 
of acute pericarditis and 7\% of cardiac tamponade are due to tuberculosis with a mortality rate of $14-40 \%$ [2]. We present a case of tuberculous pleuro-pericardial effusion with adenitis in an apparently immunocompetent clergyman.

\section{Clinical History}

A 61-year-old clergyman presented with a 3-week history of cough, fever and a 5-day history of progressive difficulty with breathing. Cough was non-productive and no associated chest pain or hemoptysis. He had a high grade fever, intermittent but no drenching night sweat. The cough and fever subsided with paracetamol, antimalarial drugs and antibiotic (Amoxycillin) given at a mission hospital. However, he later developed progressive dyspnoea. No orthopnoea, paroxysmal nocturnal dyspnoea, abdominal discomfort or change in bowel habit, early satiety or leg swelling. There was anorexia and weight loss. He was not a known diabetes mellitus or hypertensive patient; neither previously treated for tuberculosis nor on any medication for a chronic illness. He was married in a monogamous setting with children and neither smoked cigarette nor drank alcohol. A month prior to onset of the current problems, he was treated for left axillary furuncle at a mission hospital. With onset of dyspnoea, he was admitted but referred to our facility after 4 days of worsening clinical state.

On examination, he was conscious but dyspnoeic, afebrile, not pale, anicteric, acyanosed, no digital clubbing, healed scar and multiple matted lymph nodes in the left axilla and no peripheral oedema. Respiratory rate was $42 \mathrm{cpm}$ with reduced chest movement, expansion, tactile and vocal fremitus on the left lower lung zone. Pulse rate was 64bpm, regular, normal volume, blood pressure $110 / 70 \mathrm{mmHg}$, positive hepato-jugular reflux, and impalpable apex beat with $S_{1} S_{2}$ of reduced intensity. He had no hepatosplenomegaly.

Investigations revealed anaemia with ESR of $70 \mathrm{~mm} / \mathrm{hr}$, normal electrolyte and urea, liver function test, blood glucose, blood culture and serology. Echocardiography showed pericardial effusion with normal ventricular size, no right heart collapse, left ventricular ejection fraction of 50\%. Others are as shown in Figures 1-4.

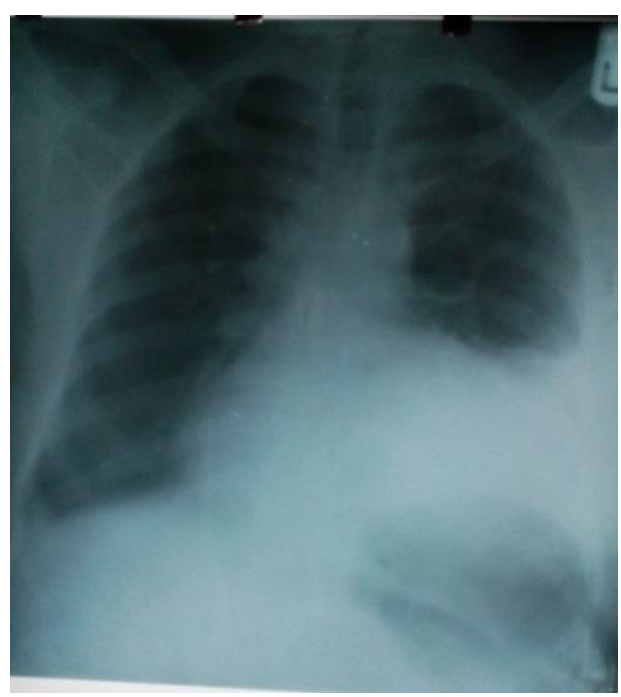

Figure 1a: Chest X-ray showing a homogeneous opacity of left lower lung zone obliterating ipsilateral cardiac border and hemidiaphragm with a meniscus sign indicative of a pleural effusion. There is double cardiac shadow suggestive of a pericardial effusion. 


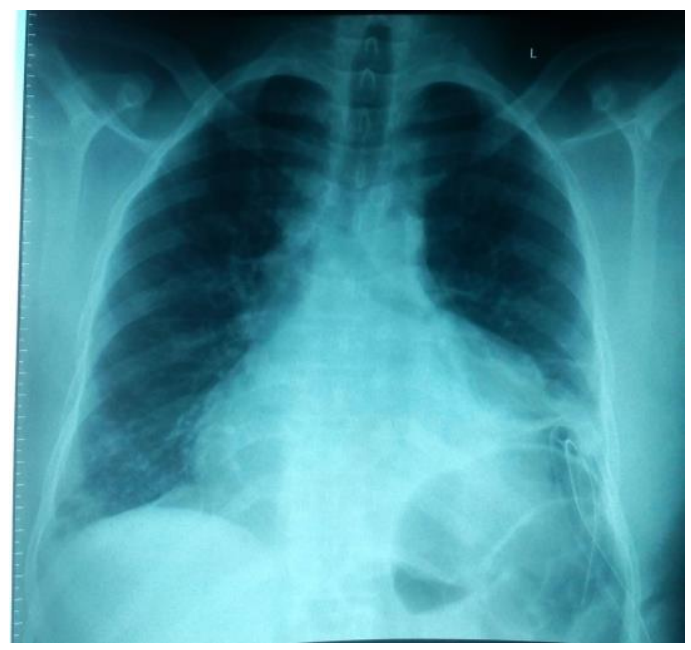

Figure 1b: Post-intubation Chest X-ray showing significant resolution of initial left lower lung homogeneous opacity. The cardiac silhouette assumes a water bottle configuration with double cardiac shadows more clearly demonstrated on the right side indicative of pericardial effusion.

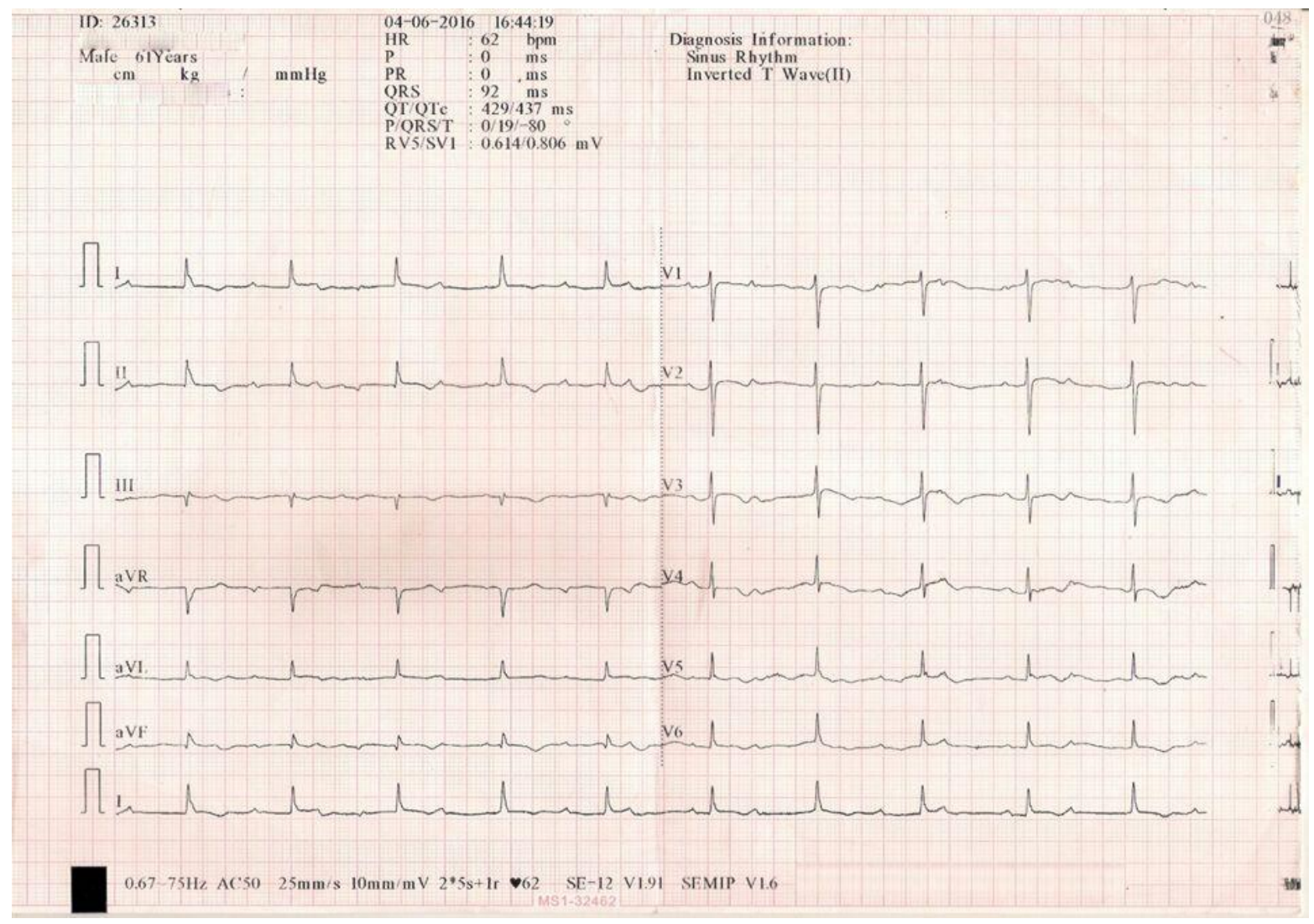

Figure 2: ECG shows $3^{\text {rd }}$ degree atrioventricular block with accelerated junctional escape rhythm (P waves march through the rhythm with a resetting of the rhythm after the $4^{\text {th }} \mathrm{p}$ wave), low voltage QRS complexes, electrical alternans, diffuse Twave inversion in all leads facing the epicardial surfaces. These are suggestive of pericardial effusion on a background of late phase of acute pericarditis. 
A

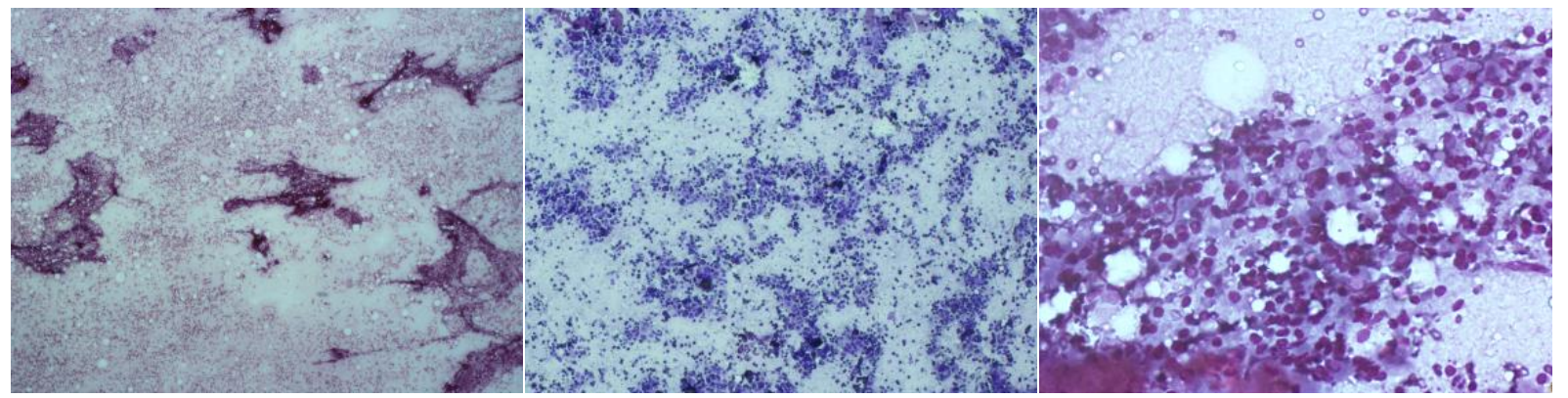

Figure 3: FNAC of Left Axillary Lymph Node: A. X 40 B. X100 C. X200: Fairly cellular aspirate with small and loose aggregate of epitheloid histiocytes with abundant cytoplasm in a background of small lymphocytes, plasma cells and dirty debris.

A

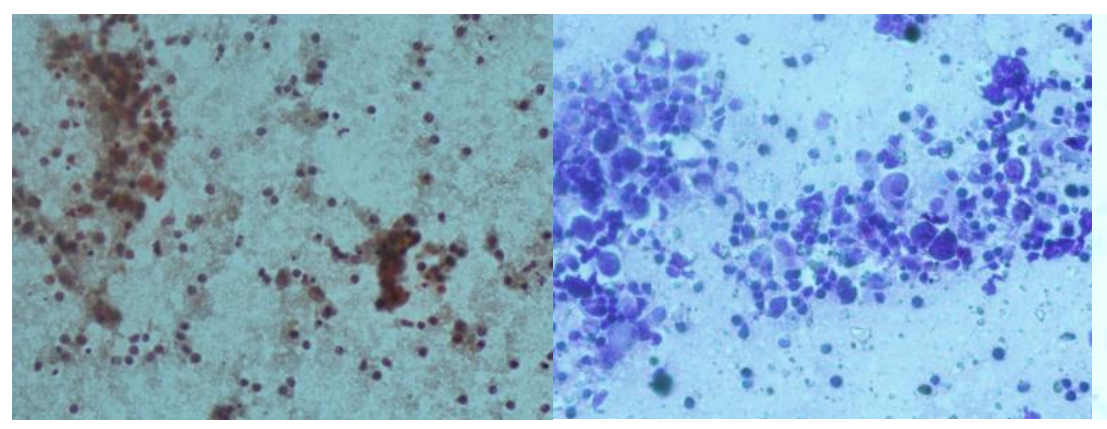

C

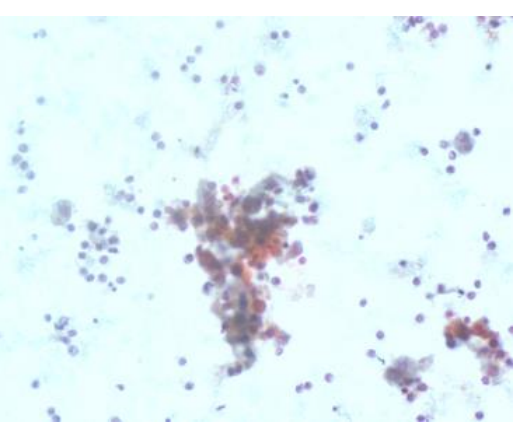

Figure 4: Left Pleural Aspirate: A. X 40 B. X100 C.X200: High proportion of lymphocytes and mesothelial cells, fibrin deposits, multi-nucleated giant macrophages and epitheloid cells in the background.

He had left-sided closed tube thoracotomy drainage of $950 \mathrm{mls}$ haemorrhagic fluid with initial subsidence of the dyspnoea. Pleural fluid and lymph node histology confirmed tuberculous chronic granulomatous inflammation. He could not be commenced on anti-tuberculous therapy as his clinical condition deteriorated suddenly and died less than 24-hour on admission despite resuscitative measures.

\section{Discussion}

Our patient had tuberculous hemorrhagic pleural effusion with concomitant pericardial effusion and left axillary adenitis. Pericardial involvement in TB which occurs in 1-8\% of cases is a clinically important TB extra-pulmonary manifestation [3]. Extra-pulmonary involvement occurs in 20\% of cases and this increases to $50 \%$ in HIV infected population [3, 4]. Pericardial spread could be from lung parenchymal, mediastinal or hilar lymph nodes, as part of miliary dissemination of the tubercle or rarely directly from tuberculous pneumonia. Presence of adenitis with serous effusion is significant. Large pericardial effusion is associated with adenitis but small effusion results from hematogenous spread [5]. 
Chest radiograph showed an enlarged cardiac silhouette suggestive of pericardial effusion with no active lung parenchymal disease. $90 \%$ of cases of tuberculous pericarditis show cardiomegaly on chest radiography but only $30 \%$ shows active lung disease according to Mayosi et al [6]. ECG revealed a $3^{\text {rd }}$ degree atrioventricular (AV) block. Nearly all cases of tuberculous pericarditis have abnormal ECG [6]. Tuberculous pericarditis is less commonly identified as a cause of $3^{\text {rd }}$ degree AV block, unlike other infectious causes like infective endocarditis, Chaga's disease, Lyme's disease. Of note was the narrow complex escape rhythm (accelerated junctional escape rhythm at a rate of $62 \mathrm{bpm})$.

The definite diagnosis of tuberculous pericarditis was by positive AFB in the pleural aspirate and typical lymph node histology, in keeping with criteria by Mayosi et al [6]. As timely intervention in tuberculous pericarditis could be life saving, its accurate diagnosis which is often difficult should be rapid [7]. This is the situation in our case as the patient presented at a hospital where he was managed for pneumonia and left axillary boils but referred late to our facility resulting in his demise even before comprehensive pathological results came out let alone commencement of anti-tuberculosis therapy.

\section{Conclusion}

TB remains one of the deadliest communicable diseases despite the availability of effective treatment and the use of a potent live attenuated vaccine. Haemorrhagic pleuro-pericardial effusion with adenitis even without any obvious immunocompromised risk factor should trigger high index of suspicion of tuberculosis, especially in endemic region like sub-Saharan Africa. This would facilitate early empirical treatment with a view to reducing over-all morbidity and mortality associated with TB.

\section{Acknowledgment}

We most especially express our gratitude to Drs A.G. Adeyeye and T.O. Ojo of Obafemi Awolowo University Teaching Hospital, Ile-Ife, Osun State for critical reading and useful suggestion during the preparation of this manuscript; Prof Jesse Otegbayo and Prof Oladapo Walker of Babcock University, Ilishan-Remo, Ogun State for their mentoring and encouragement always. No funding from individuals or bodies received for this work.

\section{Conflicts of Interest}

The authors hereby declared no conflicts of interest in the preparation of this work.

\section{References}

1. Murray JF. A century of tuberculosis. Am J Respir Crit Care Med 169 (2004): 1181-1186.

2. Wanjari K, Baradkar V, Mathur $\mathrm{M}$, et al. A case of tuberculous pericardial effusion. Indian $\mathrm{J}$ Med Microbiol 27 (2009): 75-77.

3. Suman A, Sarin JL, Grant SC, et al. A case of tuberculous pericardial effusion. Age and ageing 32 (2003): 450-452.

4. Fanning A. Tuberculosis: 6. Extrapulmonary disease. CMAJ: Canadian Medical Association Journal 160 (1999): 1597. 
5. Wani I, Singh H, Alam I, et al. Tubercular pericardial effusion: a case report. The Internet Journal of Third World Medicine 8 (2008): 1.

6. Mayosi BM, Burgess LJ, Doubell AF. Tuberculous pericarditis: Heart disease in Africa. Circulation 112 (2005): 3608-3616.

7. Reuter H, Burgess L, Van Vuuren W, et al. Diagnosing tuberculous pericarditis. Journal of the Association of Physicians 99 (2006): 827-839.

(C) (P) This article is an open access article distributed under the terms and conditions of the

BY Creative Commons Attribution (CC-BY) license 4.0 